\title{
Aspects démographiques du grand âge en Europe
}

Demographic aspects of old age

Joëlle Gaymu

\section{(2) OpenEdition}

Journals

Édition électronique

URL : http://journals.openedition.org/ress/3723

DOI : $10.4000 /$ ress.3723

ISBN : 1663-4446

ISSN : 1663-4446

Éditeur

Librairie Droz

Édition imprimée

Date de publication : 15 mai 2017

Pagination : $19-40$

ISSN : 0048-8046

Référence électronique

Joëlle Gaymu, «Aspects démographiques du grand âge en Europe », Revue européenne des sciences sociales [En ligne], 55-1 | 2017, mis en ligne le 15 mai 2020, consulté le 19 janvier 2021. URL : http:// journals.openedition.org/ress/3723; DOI : https://doi.org/10.4000/ress.3723 


\title{
ASPECTS DÉMOGRAPHIQUES DU GRAND ÂGE EN EUROPE
}

JOËLLE GAYMU

Paris, INED

gaymu@ined.fr

\begin{abstract}
Résumé. Tous les pays européens sont engagés dans un processus de vieillissement démographique. Analysant la période 1980-2040, cet article montre que ce phénomène se pose et se posera demain, en des termes différents selon les pays en raison de la diversité de leur histoire démographique. L'accent est également mis sur les inégalités de conditions de mortalité au grand âge qui, notamment, façonnent différemment le contexte conjugal à ce stade de la vie. Toutefois, dans tous les pays c'est la population la plus âgée, ayant atteint ou dépassé l'âge de 85 ans, qui augmentera le plus, évolution qui risque de nécessiter des ajustements des politiques de prise en charge de la perte d'autonomie. Par-delà ces «certitudes» démographiques, sont évoquées certaines transformations probables du vécu du grand âge liées au renouvellement des générations qui pourraient changer la nature des besoins d'assistance des futures personnes âgées.
\end{abstract}

Mots-clés: disparités européennes, mortalité, personnes âgées dépendantes, veuvage, vieillissement démographique.

\begin{abstract}
All European countries are ageing. This article describes past and futur trends (from 1980 to 2040) of this process. The ageing map of Europe has changed and will continue to do so according to the specific history of each country. It also emphazises the differences in mortality at old ages, which will shape in different ways the marital context at that very stage of life. Yet, in all countries, the number of oldest people, aged 85 and over, will increase faster and this trend might require changes in care policies for the dependent population. Beyond these demographic "certainties", the article examines some likely changes in the way old age will be experienced, most notably in relation to generational renewal, due to the renewal of cohortes, that might produce transformations in the future care needs of elders.
\end{abstract}

Keywords: ageing, dependent elder, European differences, mortality, widowhood. 
Relevant longtemps du seul domaine de la sphère privée, le cas des personnes âgées est progressivement devenu objet d'un traitement collectif dans les sociétés occidentales. Même si la majorité d'entre elles vieillissent à domicile et en bonne santé jusqu'à des âges avancés, les risques de subir des limitations d'autonomie s'accélèrent après 75 ans et une prise en charge peut alors devenir indispensable. La fraction la plus dépendante de la population âgée est devenue un enjeu de société dans la plupart des pays européens et sa probable augmentation pourrait avoir d’importantes conséquences en termes de politique publique.

Mieux que tout autre phénomène démographique, le vieillissement a un caractère largement prévisible. La structure par âge d'une population reflète, en effet, son histoire démographique sur plus d’un siècle : la fécondité décide de l'ampleur de la base de la pyramide, la mortalité dessinant, pour l'essentiel, l’allure de son sommet. Dans les pays européens, l’augmentation de la proportion de personnes âgées durant les dernières décennies tient au cumul de la chute de la fécondité et de la baisse de la mortalité aux âges avancés. Aujourd'hui, dans certains pays, les progrès ne touchent plus, pour l'essentiel, que les personnes âgées, la mortalité étant très faible de la naissance à la fin de la vie active (Monnier, 2006; Meslé et Vallin, 2002b). Quelles que soient les hypothèses de projection envisagées, le vieillissement de la population européenne est appelé à se poursuivre (Giannakouris, 2008; Goll, 2010; Eurostat, 2015), l'avancée en âge des générations nombreuses du baby boom ${ }^{1}$ étant un autre facteur d'accélération de ce processus (Monnier, 2007). En 1980, moins de $5 \%$ de la population européenne avait 75 ans et plus, la proportion était quasiment 2 fois plus élevée en 2015 et elle pourrait dépasser $16 \%$ en 2050. Tous les pays européens sont engagés dans ce processus mais à des rythmes différents, tous n’ayant pas connu la même histoire démographique. Cet article aborde ainsi, dans un premier temps, les inégalités géographiques dans la dynamique du vieillissement, les évolutions futures étant prévisibles avec une grande marge de certitude du fait de l'inertie des phénomènes démographiques. L’accent est également mis sur la diversité des conditions de mortalité aux grands âges qui 
contribue à expliquer l'inégale ampleur, actuelle et future, du vieillissement démographique des territoires européens.

Si l'on ne peut nier que cet avenir démographique appellera des adaptations des politiques de prise en charge de la dépendance, tirer des conséquences sur les futurs besoins d’aide à la lumière de la seule évolution des effectifs globaux des personnes âgées peut être simplificateur et trompeur. Ainsi, tout progrès dans le domaine de la santé contribuera à abaisser la pression de la croissance numérique de la population âgée. Or, les caractéristiques des personnes âgées résultent, certes, de leur vieillissement biologique mais aussi de leur appartenance à une génération particulière dont le parcours est susceptible de marquer les comportements. Une trentaine de générations constituent la fraction la plus âgée de notre population, autant donc d'histoires spécifiques qui, au fil du temps, changent les modes de vie au grand âge. Si, dans l'esprit collectif, cette période de la vie est généralement associée à des problèmes de santé, les évolutions passées montrent, qu'en fait, elle est de plus en plus souvent synonyme d'autonomie. Même à des âges très avancés, on vieillit désormais majoritairement chez soi, en couple ou seul (Tomassini et al., 2004; Gaymu et al., 2006).

Les personnes qui, aujourd'hui, ont 85 ans seront toutes décédées en 2050 et celles qui à cette date auront franchi cette frontière d'âge sont les quinquagénaires actuels. Avec ce renouvellement des générations, les personnes âgées de demain seront, sans nul doute, différentes de celles d'aujourd'hui sur bien des points. Cet article décrira certaines transformations prévisibles, notamment en matière d'environnement familial, qui risquent de changer le contexte de vie aux âges avancés et, par là même, le volume et la nature des besoins d’assistance des futures personnes âgées. Par-delà certains traits communs à tous les Européens âgés, on évoquera, enfin, quelques interrogations relatives à l'implication familiale et aux orientations données à la solidarité collective qui, aujourd'hui, sont à l'origine de forts contrastes dans le vécu et la prise en charge du grand âge en Europe. 


\section{LA CARTE DU VIEILLISSEMENT DE L'EUROPE A CHANGÉ}

À l'échelon de l'ensemble des 28 pays de l'Union européenne, la proportion de personnes âgées de 75 ans et plus a quasiment doublé entre 1980 et 2015 passant de 4,8 à 9\%. En 1980, l'Europe de l'Ouest et du Nord étaient les zones les plus vieillies (5,5\%), l'Europe centrale et du Sud ayant les plus faibles proportions de personnes âgées de 75 ans et plus (de l'ordre de $4 \%$ ). 35 ans plus tard, ce classement est bouleversé (cf. Tableau i) et c’est désormais l'Europe du Sud (ı, I \%) qui est la zone la plus vieillie. Elle est suivie par l’Europe de l'Ouest et les pays baltes alors que l'Europe du Nord constitue désormais avec l'Europe centrale le peloton de queue (respectivement 8 et 7,2\%).

Si l'on descend à l'échelon des pays (cf. Graphique I), en i980, la proportion de personnes âgées de 75 ans et plus égalait ou dépassait 4,5\% dans la moitié des pays Européens. Avec des valeurs proches de $6 \%$, la Suède, l'Allemagne, l'Autriche, la Norvège et la France étaient en tête. À l'autre extrême, les proportions de la plupart des pays situés en Europe méridionale et centrale étaient en dessous de la valeur médiane, certains (Roumanie, Bulgarie, Slovaquie, Pologne) comptant même moins de 3,5\% de personnes âgées de 75 ans et plus.

Tableau I. Évolution (1980-2050) de divers indicateurs de vieillissement dans les grandes régions européennes

\begin{tabular}{|c|c|c|c|c|c|c|c|c|c|}
\hline & \multicolumn{3}{|c|}{ \% DE 75 ANS ET PLUS } & \multicolumn{3}{|c|}{ \% DE 85 ANS ET PLUS } & \multicolumn{3}{|c|}{$\begin{array}{l}\text { \% } 85 \text { ANS ET PLUS, PARMI } \\
\text { LES } 75 \text { ANS ET PLUS }\end{array}$} \\
\hline & 1980 & 2015 & 2050 & 1980 & 2015 & 2050 & 1980 & 2015 & 2050 \\
\hline Europe du Nord (I) & 5,5 & 8,0 & 13,1 & 1,0 & 2,4 & 4,7 & 17,9 & 29,7 & 36,0 \\
\hline Europe de l'Ouest (2) & 5,6 & 9,2 & 16,0 & 0,9 & 2,6 & 6,1 & 16,9 & 28,3 & 38,2 \\
\hline Pays Baltes & 4,6 & 9,2 & 17,0 & 0,8 & 2,2 & 6,4 & 17,9 & 23,5 & 37,7 \\
\hline Europe centrale & 3,6 & 7,2 & 14,7 & 0,5 & ।,7 & 4,9 & 13,0 & 23,1 & 33,2 \\
\hline Europe du Sud (3) & 4,2 & 10,1 & 18,6 & 0,7 & 2,9 & 6,6 & 16,5 & 28,4 & 35,2 \\
\hline Union européenne (28) & 4,8 & 9,0 & 16,4 & 0,8 & 2,5 & 6,0 & 16,3 & 27,5 & 36,6 \\
\hline
\end{tabular}

(I) y compris Norvège et Islande; (2) y compris Suisse; (3) sans la Croatie en 1980.

Source: Eurostat (calculs de l'auteur). 
35 années plus tard, en 2015, dans tous les pays, sauf l'Irlande, la Slovaquie et Chypre, la proportion de personnes de 75 ans et plus est supérieure à la valeur maximale observée en 1980 (6,4\% en Suède). L’Allemagne occupe la tête du classement avec, désormais, l'Italie et la Grèce (environ I I \%), suivies par le Portugal (9,9\%), les trois pays baltes, l'Espagne, la France et la Belgique (environ 9\%). Dans les autres pays d'Europe septentrionale et occidentale (Royaume-Uni, Autriche, Danemark, Pays-Bas, Norvège), la proportion de personnes âgées de 75 ans et plus est désormais comparable, voire inférieure à la valeur médiane (soit 8,4\%). Autrement dit, la carte du vieillissement européen s'est modifiée et la hiérarchie des pays observée en 2015 ne correspond plus que très faiblement à celle observée 35 ans plus tôt (coefficient de corrélation de o,3). Malgré cette redistribution, il n'y a eu qu'une très légère homogénéisation des contrastes entre les pays. Globalement, le constat est le même pour l'évolution des proportions des personnes âgées de 85 ans et plus (cf. Tableau I). Zone parmi les moins vieillies il y a 35 ans, l'Europe du Sud compte en 2015 la fraction de personnes très âgées la plus élevée $(2,9 \%)$; elle est talonnée par l’Europe de l'Ouest, du Nord et de l'Est loin devant l'Europe centrale (, $7 \%$ ).

L’accélération du vieillissement dans les pays du sud de l'Europe tient au cumul d'une importante diminution de leur population jeune (liée à l'effondrement récent du niveau de leur fécondité) et d'une croissance soutenue des effectifs âgés (en raison de forts gains d'espérance de vie aux âges élevés). La plupart des pays d'Europe centrale et les trois pays baltes ont également connu un fort rétrécissement de la base de leur pyramide des âges mais le rythme de croissance de leur population plus âgée a été moins intense, en raison de conditions de mortalité moins favorables. En comparaison, dans les pays d’Europe de l'Ouest et du Nord, l'intensité de ces évolutions, tant à la base qu'au sommet des pyramides, a été beaucoup moins accentuée (Adveev et al., 20I I). 


\section{LE VIEILLISSEMENT DE LA POPULATION EUROPÉENNE VA SE POURSUIVRE}

Dans l'avenir, le vieillissement de la population européenne va se poursuivre. Selon les projections élaborées par Eurostat (Eurostat, 2015; European Commission, 2012; Giannakouris, 2008; Goll, 2010; Lanzieri, 20II ), en 2050, I6,4\% de la population de l'Union européenne pourrait avoir 75 ans et plus et ce pourcentage dépasserait $15 \%$ dans la moitié des pays (cf. Graphique I). À cette date, l'Europe du Sud serait toujours en tête (I8,6\%) devançant les pays baltes et de l'Ouest (I6 à I7\%). Désormais, c'est en Europe du Nord que la proportion de personnes âgées de 75 ans et plus serait la moins élevée (I3,I \%), inférieure à celle observée en l'Europe centrale (I4,7\%). Contrairement à la période précédente, la hiérarchie des pays devrait, globalement, rester la même (coefficient de corrélation de 0,72 ), la plupart des pays progressant au même rythme vers des niveaux de vieillissement plus élevés. Ce maintien des contrastes entre les pays est probablement favorisé par l'hypothèse de convergence ${ }^{2}$ des comportements sous-tendant ces projections européennes (European Commission, 2012 ; Giannakouris, 2008).

Graphique I. Proportions de personnes âgées de 75 ans et plus dans les pays européens en 1980, 2015 et 2050

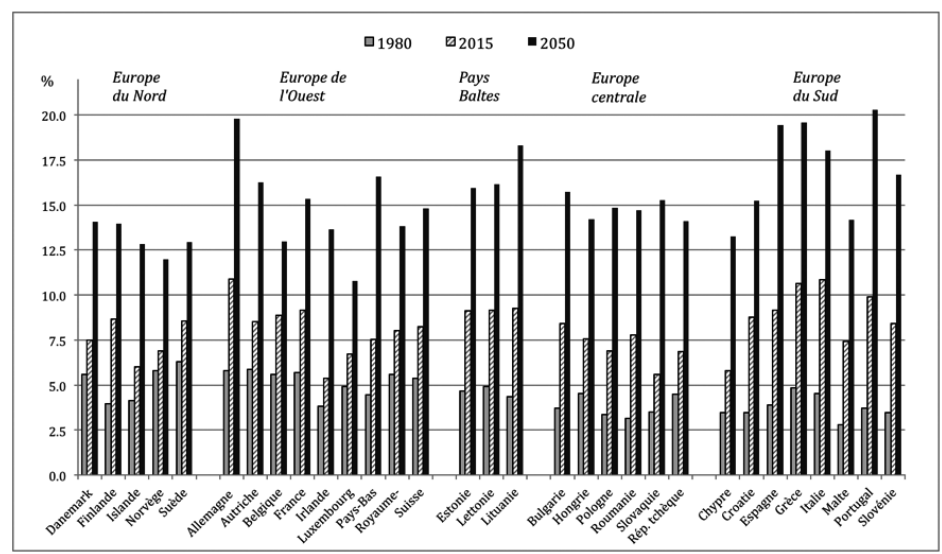

Source: Eurostat.

2 Ainsi, l'augmentation de l'espérance de vie est supposée être plus forte dans les pays ayant un plus faible niveau initial. 
Entre 2015 et 2050, la proportion de personnes âgées de 75 ans et plus pourrait être multipliée par plus de I,5 dans tous les pays (I7 étant dans ce cas durant la période précédente), aux deux exceptions près de la Belgique et la Suède. En 2050, avec un pourcentage de 20,3\%, le Portugal serait en tête, talonné par l’Allemagne, et suivis par la Grèce et l'Espagne (environ I9,5\%), l'Italie et la Lituanie (I $8 \%$ ). À l'autre extrême de l'échelle, se trouveraient toujours des pays majoritairement situés en Europe septentrionale: le Luxembourg (ı,8\%), la Norvège (1 $2 \%$ ), l’Islande, la Suède et la Belgique (environ I 3 \%).

Dans l'avenir, les populations jeune et adulte devraient décroître dans la quasi-totalité des pays d'Europe du Sud, du centre et baltes. A contrario, dans la plupart de ceux d'Europe de l'Ouest et du Nord ${ }^{3}$, ces populations pourraient stagner ou même augmenter (Norvège, Suède, Suisse, Luxembourg, Belgique) (Adveev et al., 20 I ). Parallèlement, dans tous les pays, à quelques exceptions près, la population âgée de 75 ans et plus continuerait de croître à un rythme aussi soutenu, voire supérieur, que celui observé entre i980 et 20 I5 (cf. Tableau 2) : 7 pays sont susceptibles de connaître deux doublements successifs de ces effectifs (Pays-Bas, Luxembourg, Islande, Finlande, Roumanie, Malte, Espagne).

Tableau 2. Évolution des effectifs de personnes âgées de 75 ans et plus dans les pays européens entre 1980 et 2015 et entre 2015 et 2050

\begin{tabular}{|c|c|c|c|}
\hline \multirow{2}{*}{$\begin{array}{c}1980-2015 \\
(1980=100)\end{array}$} & \multicolumn{3}{|c|}{$2015-2050(2015=100)$} \\
\cline { 2 - 4 } $129-175$ & Estonie, Lettonie & $\begin{array}{c}\text { Danemark, Suède, Norvège, } \\
\text { Autriche, Hongrie, } \\
\text { République Tchèque }\end{array}$ & $\begin{array}{c}\text { 200-357 } \\
\text { Royaume-Uni } \\
\text { Slovaquie }\end{array}$ \\
\hline $176-199$ & Suisse & Belgique, Lituanie, Bulgarie & Allemagne, France, Irlande \\
$200-357$ & Grèce, Pologne, & Italie, Portugal & $\begin{array}{c}\text { Islande, Finlande, } \\
\text { Luxembourg, Pays-Bas, } \\
\text { Roumanie, Espagne, Malte, }\end{array}$ \\
\hline & Croatie & Slovénie & Chypre \\
\hline
\end{tabular}

Source: Eurostat (calculs de l'auteur). 
L'évolution de ces nombres risque de jouer sur la politique de la prise en charge de la perte d’autonomie d'autant plus que, dans les années futures, les effectifs des personnes les plus âgées vont augmenter le plus vite. Or, elles souffrent généralement d’une multiplicité de problèmes de santé et/ou vivent dans un environnement social moins protecteur (réseaux sociaux plus restreints, composés de membres plus âgés), ce qui accroît leurs besoins de soutien. Entre 2015 et 2050, la population de l'Union européenne âgée de 85 ans et plus est susceptible d'être multipliée par 2,5 (contre 1,64 pour celle âgée de 75 à 84 ans). La croissance serait la plus faible dans les pays baltes et la plus forte dans certains pays d'Europe septentrionale (Islande, Norvège, Danemark, Pays-Bas, Irlande) et en Suisse où ces effectifs pourraient tripler ou quasiment. Autrement dit, dans l'avenir la population âgée sera composée de personnes de plus en plus âgées : en 2015, 26,5\% de celles âgées de 75 ans et plus avaient fêté leur $85^{\mathrm{e}}$ anniversaire, elles pourraient être $36 \%$ en 2050. À cette date, les proportions les plus élevées s'observeraient, pour l'essentiel, en Europe de l'Ouest (Allemagne, France) et du Nord (Finlande, Islande). L’avancée en âge des générations du baby boom et la poursuite de la baisse de la mortalité dans les hypothèses de projection conjuguent leurs effets pour expliquer cette tendance.

L’inégal degré de vieillissement, présent et à venir, des grandes régions européennes est en partie modelé par l'existence de fortes disparités en matière de mortalité. Au cours des dernières décennies, atteindre le grand âge est devenu plus fréquent et cette période de la vie s’est allongée dans tous les pays mais le rythme de ces améliorations n’a pas été le même partout.

4 Ces coefficients multiplicateurs atteignant respectivement 2,8 et 1,7 avec une hypothèse de baisse de la mortalité plus forte. 


\section{LA PERSISTANCE DE FORTES INÉGALITÉS DE MORTALITÉ AUX GRANDS ÂGES}

Depuis les années 1970, les progrès effectués en matière de lutte contre les maladies, notamment cardio-vasculaires, se sont traduits par une accélération du recul de la mortalité aux âges avancés dans tous les pays européens. Toutefois, les pays de l'Est sont restés en marge de la marche vers la réduction de la mortalité cardiovasculaire, puis la crise, la dégradation de leur système de santé publique, l'alcoolisation de la population, en particulier masculine, ont marqués les deux décennies suivantes et même si, depuis la fin des années 1990, l'espérance de vie augmente à nouveau, le retard vis-à-vis du reste de l'Europe ne change presque pas (Meslé et Vallin, 2002a; Monnier 2006; Avdeev et al, 20I I).

Le nombre de survivants à 75 ans illustre clairement ces contrastes. En Europe de l'Ouest, du Nord et du Sud, fêter son $75^{\mathrm{e}}$ anniversaire est devenu presque banal pour les femmes : tel pourrait être le cas de plus de $80 \%$ des Italiennes, Françaises ou Suédoises, nées en 2008 (environ $70 \%$ des hommes). À l'autre extrémité de l'échelle, seuls environ $40 \%$ des hommes hongrois, bulgares ou estoniens pourraient atteindre cet âge (contre $70 \%$ des femmes). Les inégalités d'espérance de vie à 75 ans sont également très fortes et ont, de surcroit, augmenté.

Entre 1980 et 20I4, côté masculin, les pays d'Europe de l'Ouest et du Sud ont enregistré les progrès les plus rapides (soit une augmentation de la durée de vie de 3,7 ans en moyenne, cf. Tableau 3), comblant ainsi l'avance prise par ceux d'Europe du Nord: en 20I4, l'espérance de vie à 75 ans est du même ordre de grandeur dans ces trois régions (environ II,5 ans). L’Europe du Sud est, toutefois, une terre contrastée : en Espagne, Grèce et Italie les hommes de 75 ans peuvent espérer vivre aussi longtemps que leurs homologues d’Europe de l'Ouest ou du Nord, alors dans les autres pays la mortalité se situe juste dans la moyenne européenne, voire en dessous. En 1980, tous les pays d’Europe centrale, et dans une moindre mesure les pays Baltes, étaient en retard par rapport à la moyenne européenne ; 30 ans plus tard, ils sont encore plus distancés. C'est dans cette zone que l'augmentation de la durée de vie des hommes a été la plus faible (en moyenne de I,7 ans dans les pays baltes et 2,4 ans en Europe centrale). On prend ici la mesure du retard accumulé : l'espérance de 
vie à 75 ans de ces pays est du même ordre de grandeur que celle atteinte par la plupart des pays scandinaves en 1980, soit environ 9 ans, avec un minimum de 8,7 ans en Bulgarie. Seule la Pologne échappe à cette tendance et se situe proche de la moyenne européenne.

Tableau 3. Espérances de vie à 75 ans (en années) selon le sexe dans les grandes régions européennes: évolution entre 1980 et 2014.

\begin{tabular}{|l|c|c|c|c|c|c|c|c|}
\hline & \multicolumn{3}{|c|}{ HOMMES } & \multicolumn{3}{c|}{ FEMMES } & \multicolumn{2}{c|}{ FEMMES-HOMMES } \\
\cline { 2 - 11 } & 1980 & 2014 & $2014-1980$ & 1980 & 2014 & $2014-1980$ & 1980 & 2014 \\
\hline Europe du Nord & 8,5 & 11,4 & 3,0 & 10,9 & 13,5 & 2,7 & 2,4 & 2,1 \\
\hline Europe de l'Ouest & 7,8 & 11,6 & 3,7 & 10,0 & 13,9 & 3,9 & 2,2 & 2,3 \\
\hline Pays Baltes & 7,6 & 9,3 & 1,7 & 9,4 & 12,2 & 2,9 & 1,8 & 2,9 \\
\hline Europe Centrale & 7,0 & 9,4 & 2,4 & 8,5 & 11,5 & 3,0 & 1,5 & 2,1 \\
\hline Europe du Sud & 7,6 & 11,3 & 3,7 & 9,1 & 13,5 & 4,4 & 1,5 & 2,2 \\
\hline Ensemble & 7,7 & 10,8 & 3,1 & 9,6 & 13,1 & 3,5 & 1,9 & 2,3 \\
\hline
\end{tabular}

Source: Human lifetable et Eurostat (calculs de l'auteur).

Chez les femmes, en 2014, c'est également dans les pays baltes et en Europe centrale que les durées de vie à 75 ans sont les plus courtes (respectivement I2,2 et II,5 ans) et en Europe de l'Ouest, du Sud et du Nord qu'elles sont les plus longues (de l'ordre de I3,5 ans en moyenne). Durant les 30 dernières années, chez elles, contrairement aux évolutions masculines, les gains enregistrés en Europe du Nord ont été les plus faibles (2,7 ans), comparables à ceux des pays baltes et de l'Est, mais très inférieurs à ceux observés en Europe méridionale ( 4,4 ans) et de l'Ouest (3,9 ans). La durée de vie à 75 ans des femmes scandinaves (hormis les Islandaises), pour la plupart les plus élevées en 1980, est désormais inférieure d'une année à celle des Italiennes, Espagnoles, Françaises ou Suisses.

La diversité de ces tendances s'est répercutée sur la surmortalité masculine, qui tant en termes de niveaux que d'évolutions, différencie fortement les pays européens. 


\section{UN DÉBUT DE CONVERGENCE ENTRE LES ESPÉRANCES DE VIE DES HOMMES ET DES FEMMES À 75 ANS?}

Durant les dernières décennies, à la naissance (Meslé, 2006) comme à 65 ans (Thorslund et al., 20I3), un processus de convergence des espérances de vie des hommes et des femmes s'est progressivement diffusé du Nord au Sud de l'Europe, les pays septentrionaux ayant connu une baisse plus précoce de la surmortalité des hommes dans certaines causes de décès, en particulier cardiovasculaire et cancéreuse. Dans certains pays, le recul chez les hommes de certaines attitudes nocives pour leur santé se double, plus récemment, de leur diffusion chez les femmes. Ainsi, le ralentissement de l'amélioration de la durée de vie des femmes du Nord, en comparaison notamment de celles du Sud, pourrait être dû à leur surconsommation de tabac (Vollset, 2008).

On retrouve à 75 ans une amorce de cette convergence. Si pour l'ensemble des pays européens, l'écart entre l'espérance de vie à 75 ans des hommes et des femmes s'est légèrement accentuée, (2,3 ans en 20I4 contre 1,9 an en 1980), il s'est réduit en Europe du Nord, est resté quasiment inchangé à l'Ouest et s'est creusé dans le reste de l'Europe (cf. Tableau 3). Dans tous les pays d'Europe du Nord, au Royaume-Uni et aux Pays-Bas, la durée de vie a progressé moins vite chez les femmes que chez les hommes. Dans les autres pays d'Europe de l'Ouest l'avantage des femmes s'est stabilisé, sauf en France et au Luxembourg où il a progressé d'une demi-année. Ailleurs, les écarts se sont également légèrement creusés et il n'y a plus guère que dans certains pays baltes (Estonie, Lituanie) et du Sud (Espagne, Slovénie) que l'espérance de vie des femmes à 75 ans a continué à augmenter beaucoup plus vite que celle des hommes. Dans ces pays, les écarts sont les plus forts, soit de l'ordre de 3 ans en 2014.

Toutefois, l'espérance de vie est un indicateur imparfait de l'état sanitaire d'une population, un niveau élevé ne signifiant pas nécessairement un meilleur état de santé. Ainsi, partout en Europe, les femmes vivent plus longtemps que les hommes, mais passé 65 ans, à âge égal, elles souffrent plus souvent qu'eux d'incapacités et ce, quel que soit le niveau de sévérité (Cambois et al., 2003; Van Oyen et al., 2oıо). En outre, la dispersion géographique des durées de vie 
avec ou sans incapacités ${ }^{5}$ est très forte. Ainsi, dans la plupart des pays d'Europe du Nord et de l'Ouest, les femmes de 65 ans peuvent espérer vivre I9 à 20 années sans incapacités alors que dans les pays baltes et la plupart de ceux d'Europe centrale cette durée est de l'ordre de io ans. On trouve ici une illustration supplémentaire du fossé séparant le Nord et l'Ouest, dans la meilleure situation en termes d'espérance de vie en bonne santé, et l'est et le centre de l'Europe dans la plus défavorable, le Sud occupant une position intermédiaire.

Ces inégalités ont aussi des conséquences sur le contexte conjugal du vieillissement. En cas de perte d'autonomie, un soutien au quotidien est souvent indispensable et l'on sait que, dans ce domaine, partout en Europe, les conjoints jouent un rôle essentiel (Fontaine et al., 2007). Or, compte tenu des différences de niveaux de mortalité et surmortalité masculine, les risques de connaître le veuvage varient fortement selon les pays, même si, partout, cette situation est beaucoup plus courante pour les femmes que pour les hommes.

\section{DES INÉGALITÉS PERSISTANTES, SELON LES PAYS ET LE SEXE, EN MATIĖRE DE SITUATION CONJUGALE}

Dans les générations actuelles de personnes âgées, la surmortalité masculine, l'écart d'âge au mariage et les plus fortes chances de remise en union des hommes conjuguent leurs effets dans le sens d'un très fort veuvage féminin alors que les hommes restent généralement mariés jusqu’à leur décès. De plus, les femmes survivent plus longtemps que les hommes dans le veuvage : il y a une surmortalité des veufs par rapport aux mariés mais elle est plus forte chez les hommes (Murphy et al., 2007).

Le décalage entre situation matrimoniale masculine et féminine est, de ce fait, très accusé. Par exemple, à 75-84 ans, les trois quart des hommes européens sont mariés (35\% des femmes) alors que la majorité des femmes sont veuves (17\% des hommes) (cf. Graphique 2). Ce décalage entre situations masculines et féminines

5 Signalons que, malgré les efforts d'harmonisation des données, cette dispersion peut, en partie, être liée à des différences d'appréciation, selon le contexte socioculturel, de la sévérité des incapacités. 
s'observe dans tous les pays mais dans les pays baltes et d'Europe centrale le risque de veuvage est plus élevé, conséquence de hauts niveaux de mortalité et de surmortalité masculine: dans ces zones, à 75-84 ans, moins du quart des femmes sont mariées et autant d'hommes sont déjà veufs, soit des niveaux du même ordre de grandeur que ceux observés 20 ans plus tôt en Europe de l'Ouest.

Graphique 2. Structure matrimoniale (en \%) des personnes âgées selon l'âge et le sexe dans les grandes régions européennes en $201 \mathrm{I}$
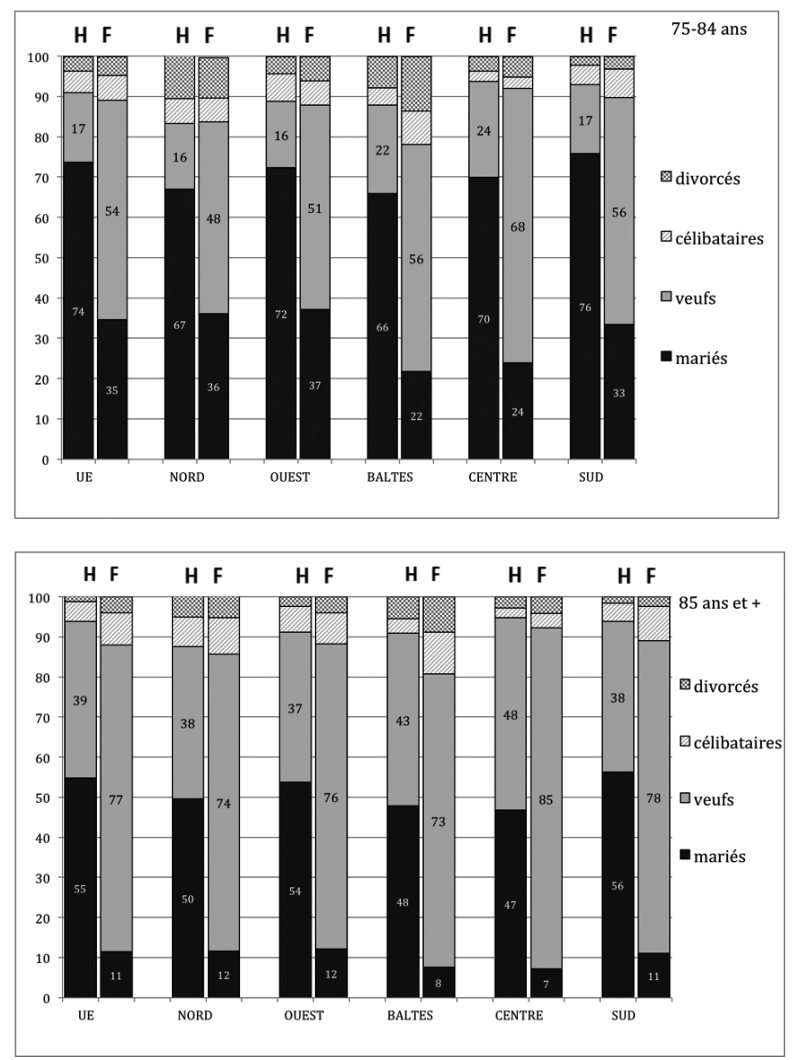

Note: dans l'avenir davantage de personnes âgées vieilliront en couple. 
Durant les dernières décennies, l'amélioration des conditions de mortalité a réduit le risque de veuvage précoce conduisant à une prolongation de la durée de vie en couple (Pennec et Gaymu, 20I I). Ainsi, selon les recensements, dans les pays d'Europe de l'Ouest par exemple, en I99i entre $16 \%$ (Allemagne) et $22 \%$ (France) des femmes de 75 ans et plus étaient mariées; en 20 I ces proportions dépassent partout 30\%. Avec la baisse attendue de la mortalité, cette tendance devrait se poursuivre dans l'avenir mais, l'arrivée aux grands âges de générations ayant davantage divorcé devrait, à l'inverse, accroître l'isolement conjugal. Chez les femmes, la première tendance devrait l'emporter et dans l'avenir, partout en Europe, elles franchiront plus fréquemment les étapes de la vieillesse en couple (Keilman et Christiansen, 20ı ; Kalogirou et Murphy, 2006 ). Selon certaines projections (Gaymu et al., 2007) en 2030, environ $40 \%$ de celles souffrant d'incapacités et âgées de 75-84 ans pourraient être en union (contre $25 \%$ en moyenne en 2000). Les proportions pourraient augmenter plus fortement encore chez les femmes de 85 ans et plus, passant de 9 à $22 \%$ en moyenne et triplant dans la plupart des pays d'Europe de l'Ouest et du Nord. Vieillir à deux sera également plus fréquent pour les hommes dépendants âgés de 85 ans et plus (48\% en 2030 contre $38 \%$ en 2000), les évolutions étant moins nettes en deçà de cet âge ${ }^{6}$.

Autrement dit, dans l'avenir, le grand âge devrait être plus fréquemment vécu en couple. Or, par-delà le soutien affectif du conjoint, les effets bénéfiques de la vie à deux sont nombreux et communs aux personnes âgées quel que soit leur pays d’appartenance. Elle a, notamment, un effet protecteur sur la mortalité et l'état de santé, améliore la situation économique, l'intégration sociale et la satisfaction de la vie (Biro, 20I3; Schaan, 20ı3; Gaymu et Springer, 2012). Par ailleurs, en tant que pourvoyeur d'aide, le conjoint permet de retarder voire d'éviter l'entrée en institution; la faible proportion des personnes mariées hébergées en institution en est l'illustration (Gaymu et al., 2006).

6 À 75-84 ans, rares sont les hommes veufs et la baisse du risque de veuvage ne devrait pas, partout, compenser la croissance de la proportion de divorcés, tendances dans la lignée de celles observées durant les dernières décennies en deçà de 75 ans. 


\section{UNE GRANDE DIVERSITÉ DE SITUATIONS RÉSIDENTIELLES AU GRAND ÂGE}

Quand l'indépendance résidentielle vient à manquer, l'institutionnalisation et la cohabitation multigénérationnelle apparaissent comme des solutions alternatives et le contexte géographique modèle fortement ces modalités de l'accompagnement des personnes en perte d'autonomie. Le recours à l'institutionnalisation en est un exemple. Ainsi, chez les femmes âgées de 85 ans et plus, ce mode de vie est près de 4 fois plus fréquent dans les pays du Nord et de l'Ouest de l'Europe qu'ailleurs (soit de l'ordre de $20 \%$ contre $5 \%$ en Europe centrale ou de l'Est et $8 \%$ dans le Sud). Selon les pays, les pourcentages varient de i à Io: en Bulgarie ou Hongrie moins de $2 \%$ des femmes de 85 ans et plus vivent en institution alors que cette proportion est de l'ordre de $25 \%$ en Belgique, France, Irlande ou aux Pays-Bas. Au contraire des pays du nord-ouest de l'Europe où ce mode de vie est développé, dans ceux du sud et de l'est la cohabitation multigénérationnelle est privilégiée (Fontaine et al., 2007; Mönkediek et Bras, 20I4). Toutefois, le comportement des familles et le contexte politique sont inséparables et interdépendants. Par exemple, en dehors de la question des coûts, le faible développement de structures pour accueillir les personnes âgées en Europe du Sud saccommode sans conteste de la tradition d'une forte implication familiale. Mais à l'inverse, le maintien d'une fréquente co-résidence multigénérationnelle dans la région ne reflète-t-il pas le manque d’alternatives?

Quoique les contrastes entre les pays restent marqués, durant les dernières décennies partout l'autonomie résidentielle s'est accrue au grand âge : outre la vie en couple qui se prolonge, la cohabitation multigénérationnelle est moins fréquente et l'entrée en institution plus tardive (Tomassini et al, 2004). Ces tendances s'expliquent, en partie, par l'amélioration de la situation socioéconomique des retraités qui, sur certains points, pourrait se poursuivre. En effet, en cas d'incapacités, les personnes âgées de demain devraient être mieux armées que celles d'aujourd'hui, tant sur un plan personnel (parce que plus instruites) que socialement (car plus fréquemment soutenues par leur conjoint) ou économiquement (en raison de la multiplication du nombre de couples où chacun disposera d'une retraite). Par-delà l'évolution positive de ces caracté- 
ristiques individuelles, le sort des futures personnes âgées dépendra aussi - et peut être surtout - du désir et des possibilités d’aide exprimés et offerts par les familles, ainsi que des futures politiques publiques, la complémentarité entre soutien des proches et aide publique jouant un rôle majeur dans le maintien à domicile des personnes en perte d'autonomie (Attias-Donfut et Ogg, 2009).

\section{CERTITUDES DÉMOGRAPHIQUES ET INCERTITUDES COMPORTEMENTALES}

Cette incursion dans l'Europe du grand âge montre que le vieillissement de la population se pose et se posera demain, en des termes différents selon les pays en raison de la diversité de leurs histoires démographiques. Zone parmi les moins vieillies il y a 35 ans, l'Europe du Sud comptera en 2050 la fraction de personnes âgées de 75 ans et plus la plus élevée (environ 19\%); elle sera talonnée par l'Europe de l'Ouest et les pays baltes, loin devant l'Europe centrale et du Nord (13\%). Dans tous les pays, c'est la population la plus âgée, ayant atteint ou dépassé l'âge de 85 ans, qui augmentera le plus; elle pourrait tripler dans certains pays d'Europe de l'Ouest et du Nord. Même si les projections démographiques prévoient une convergence des conditions de mortalité aux grands âges, les disparités restent actuellement marquées entre l'Est et l'Ouest. Elles façonnent différemment le contexte conjugal du grand âge, conduisant davantage de personnes à vieillir en couple en Europe du Nord, de l'Ouest et du Sud. De même, actuellement, le risque de vieillir en mauvaise santé varie fortement selon les pays; le Nord et l'Ouest, étant dans la meilleure situation en termes d'espérance de vie en bonne santé, l'Est et le centre de l'Europe dans la plus défavorable, le Sud occupant une position intermédiaire.

Malgré cette diversité des territoires européens, on peut penser que les tendances communes dessinées par la démographie nécessiteront des ajustements des politiques de prise en charge de la perte d'autonomie. Ces «certitudes» démographiques ne décident, toutefois, pas de tout et il existe de nombreuses interrogations sur les transformations du vécu du grand âge liées au renouvellement des générations. Ainsi, l’accroissement des besoins de soins pourrait se révéler moins important que ne le suggèrent les indicateurs démo- 
graphiques relatifs au vieillissement, si la santé des personnes âgées s'améliorait au fil du temps. Or, certaines modifications structurelles de la population âgée (élévation du niveau d'éducation, moindre pénibilité des professions) ou changements de comportements (attitude de prévention, meilleure alimentation et hygiène de vie) peuvent laisser penser que les personnes âgées de demain seront en meilleure santé que celles d’aujourd'hui (Batljan et al., 2009). Le rôle du progrès technique, de la recherche ou encore des politiques de prévention peuvent également influencer l'évolution future de la dépendance. À l'inverse, le creusement des inégalités d’accès aux soins, le développement de l'obésité, de l'arthrose ou du diabète pourraient augmenter la probabilité d'entrer en dépendance. Aucune tendance claire ne se dégageant du passé (Jagger, 20I5), et compte tenu de la multiplicité des facteurs en jeu, l'incertitude reste de rigueur sur l'évolution future de la population dépendante, ses évaluations étant d'une large amplitude (Lecroart, 20I3).

En outre, l'équilibre entre besoins de soins formels et informels, en particulier l'institutionnalisation, risque de changer en raison des transformations de la situation familiale des personnes âgées, la forme d'assistance qu'elles reçoivent y étant intimement liée. Ainsi, en France $69 \%$ des personnes âgées dépendantes habitant seules ont recours à l'aide d'un professionnel contre $39 \%$ de celles vivant en couple ou avec d'autres personnes (Soullier et Weber, 20II). Dans le prolongement des tendances passées, certains changements positifs de l'environnement familial à ce stade de l'existence devraient se poursuivre. Ainsi, la baisse de la mortalité et, dans certains pays septentrionaux, de la surmortalité masculine devraient conduire davantage d'hommes et de femmes à vieillir en couple chez eux et, par là même, à différer, voire éviter, l'entrée en institution. Une plus large fraction de personnes très âgées bénéficiera donc des nombreux effets positifs de la vie à deux. Toutefois, cette baisse de l'isolement conjugal, en particulier des femmes, ne se doublera d'une meilleure qualité du vécu de leur éventuelle perte d’autonomie que si leurs conjoints et enfants font face. Partout en Europe, l'implication de la famille est, aujourd'hui, très forte, même si elle s'exprime sous des formes différentes, la corésidence des générations étant privilégiée au sud et à l'est de l’Europe et les 
aides des proches non-cohabitants au nord (Fontaine et al., 2007). Le problème de la future disponibilité de cet entourage, de sa capacité ou volonté à faire face à la perte d'autonomie de leurs proches, reste entier. Ainsi, dans l'avenir, les femmes, à qui incombent plus fréquemment les soins aux parents âgés (Haberkern et al., 20I5), devraient être moins disponibles du fait de leur plus forte participation au marché du travail; moins focalisée sur la vie familiale et appartenant à des couples plus égalitaires, sans doute seront-elles plus réticentes que leurs mères à accepter les contraintes imposées par la dépendance de leurs parents et/ou beaux parents. En outre, certains émettent l'hypothèse que les aînés de demain pourraient être moins enclins à se percevoir comme dépendant de leur famille et à concevoir leurs enfants dans ce rôle d'aidant.

\section{CONCLUSION}

Aujourd'hui, dans les pays où les services professionnels sont suffisamment développés, la préférence des personnes âgées va nettement vers cette solution et ce d'autant plus qu'ils ont besoin de soins personnels ou de longue durée. Ailleurs, les attentes dans ce domaine ne semblent pas satisfaites et, partout, la majorité de l'opinion s'accorde à penser que l'essentiel de l'accompagnement de la perte d'autonomie devrait être d'ordre professionnel. Ainsi, pour $56 \%$ des Français la prise en charge des personnes âgées dépendantes doit principalement être du ressort des pouvoirs publics, la part de ceux considérant que c'est le rôle de la famille n’ayant cessé de diminuer au cours des dernières années pour atteindre environ $20 \%$ (Weber, 2006). Le rééquilibrage des rôles familiaux et professionnels passe prioritairement par le renforcement de l'offre et de l'accès aux services d'aide au maintien à domicile. À ce titre, la valorisation des métiers liés à l’aide professionnelle, largement féminisées, et la question du niveau futur des pensions de retraite sont des enjeux importants. La capacité financière des individus à faire appel à ces aides dépendra, notamment, de leurs propres ressources. Or même si les prochaines cohortes de retraités - en particulier les femmes - auront acquis des droits à retraite plus larges qu'aujourd'hui, il existe une forte incertitude dans toute l'Europe sur le futur financement des pensions de retraite. 
Les évolutions démographiques prévisibles et interrogations sur les comportements des hommes et femmes de demain confrontés à la perte d'autonomie d'un de leur proche, soulignent la nécessité de renforcer les politiques d’aides aux personnes en perte d'autonomie. Dans le cas contraire, il s'ensuivrait une pression supplémentaire sur les familles alors même qu'elles assument déjà aujourd'hui l'essentiel de la solidarité à l'égard des plus fragiles.

\section{BIBLIOGRAPHIE}

ATTIAS DONFUT Cl. et OGG J., 2009, «Évolution des transferts intergénérationnels: vers un modèle européen?», Retraite et Société, 58, p. II-29.

AVDEEV A., ERENENKO T., FESTY P., GAYMU J., LEBOUTEILLEC N. et SPRINGER S., 2011, «Populations and Demographic Trends of European Countries, 1980-2010», Population-E, 66-I, p.9-I30.

BATLJAN I., LAGERGREN M. et THORSLUND M., 2009, «Population Ageing in Sweden: the Effect of Change in Educational Composition on the Future Number of Older People Suffering Severe III-Health», European Journal of Ageing, 6, p. 20I-2II. BíRÓ A., 2013, «Adverse Effects of Widowhood in Europe», Advances in Life Course Research, I8-I, p. 68-82.

CAMBOIS E., DÉSESQUELLES A. et RAVAUD J.-F., 2003, «Femmes et hommes ne sont pas égaux face aux handicaps», Population et Sociétés, Bulletin d'information, Ined, 386.

EUROPEAN COMMISSION, 2012, The 2012 Ageing Report: Underlying assumptions and Projection Methodologie, European economy, 4/2011.

EUROSTAT, 2015, People in the EU: Who Are We and How Do We Live?, Statistical books, Luxembourg, Publications Office of the European Union.

FONTAINE R., GRAMAIN A. et WITTWER J., 2007, «Les configurations d'aide familiales mobilisées autour des personnes âgées dépendantes en Europe», Economie et Statistique, 403-404, p.97-115. 
GAYMU J., DELBÈS Ch., SPRINGER S., BINET A., DESESQUELLES A., KALOGIROU S. et ZIEGLER U., 2006, «Determinants of the Living Arrangements of Older People in Europe», European Journal of population, 22-3, p. 241-262.

GAYMU J., EKAMPER P. et BEETS G., 2007, «Qui prendra en charge les Européens âgés dépendants en 2030?», Population, 62-4, p. 789-820.

GAYMU J. et SPRINGER S., 2012, «How Does Living Alone or with a Partner Influence Life Satisfaction among Older Men and Women in Europe?», Population-E, 67-I, p. 43-70.

GIANNAKOURIS K., 2008, «Ageing Characterises the Demographic Perspectives of the European Societies», Statistics in Focus, Eurostat, 72.

GOLL M., 2010, «Ageing in the European Union: Where Exactly? », Statistics in Focus, 26/2010, Eurostat.

HABERKERN K., SCHMID T. et SZYDLIK M., 20I5, «Gender Differences in Intergenerational Care in European Welfare States», Ageing and Society, 35-2, p. 298-320.

JAGGER C., 2015, «Trends in Life Expectancy and Healthy Life Expectancy», Foresight, Government Office for Science.

KALOGIROU S. et MURPHY M., 2006, «Marital Status of People Aged 75 and Over in Nine EU Countries in The Period 2000-2030», European Journal of Ageing, 3, p. 74-81.

KEILMAN N. et CHRISTIANSEN S., 20I0, «Norwegian Elderly Less Likely to Live Alone in the Future», European Journal of Ageing, 26, p. 47-72.

LANZIERI G., 20II, «The Greying of the Baby Boomers-A Century-Long View of Ageing in European Populations», Statistics in Focus, Eurostat, 23/2011.

LECROART A., FROMENT O., MARBOT Cl. et ROY D., 2013 «Projection des populations dépendantes», Dossiers Solidarité et Santé, 43.

MESLÉ F. et VALLIN J., 2002a, «Mortalité en Europe: la divergence Est-Ouest», Population, 57-I, p. I7I-2I2. 
-, 2002b, «La transition sanitaire: tendances et perspectives», in G. Caselli, J. Vallin et G. Wunsch (dir.), Démographie: analyse et synthèse III Les déterminants de la mortalité, Paris, Ined, p.439-46I.

MESLÉ F., 2006, «Progrès récents de l'espérance de vie en France: les hommes comblent une partie de leur retard», Population, 61-4, p.437-462.

MÖNKEDIEK B. et BRAS H., 2014, «Strong and Weak Family Ties Revisited: Reconsidering European Family Structures from a Network Perspective », The History of the Family, 19-2, p. 235-259.

MONNIER A., 2006, Démographie contemporaine de l'Europe, Evolutions, tendances, défis, Paris, Armand Colin.

-, 2007, «Le Baby-Boom, suite et fin», Population et Sociétés, Bulletin d'information, Ined, 43I.

MURPHY M., GRUNDY E. et KALOGIROU S., 2007, «The Increase in Marital Status Differences in Mortality up to the Oldest Age in Seven European Countries, 1990-1999», Population Studies, 61-3, p. 287-298.

PENNEC S. et GAYMU J., 20II, «La durée de l'isolement conjugal et de la vie en couple chez les personnes âgées en France: quelles évolutions entre hommes et femmes au fil des générations?», Cahiers Québécois de Démographie, 40-2, p. 175-207.

REES P., VAN DER GAAG N., DE BEER J. et HEINS F., 2012, «European Regional Populations: Current Trends, Future Pathways, and Policy Options», European Journal of Ageing, 28, p. 385-416.

SCHAAN B., 2013, «Widowhood and Depression Among Older Europeans-The Role of Gender, Caregiving, Marital Quality, and Regional Context», Journals of Gerontology, Series B: Psychological Sciences and Social Sciences, 68-3, p.431-442.

SOULLIER N. et WEBER A., 20II «L'implication de l'entourage et des professionnels auprès des personnes âgées à domicile», DREES, Études et Résultats, 771.

THORSLUND M., WASTESSON J. W., AGAHI N., LAGERGREN M. et PARKERM.G., 2013, «The Rise And Fall of Women's Advantage: A Comparison of National Trends in Life Expectancy at Age 65 Years», European Journal of Ageing, 10, p.27I-277. 
TOMASSINI C., GLASER K., WOLF D.A., BROESE VAN GROENOU M.I., et GRUNDY E., 2004, «Living Arrangements Among Older People: An Overview of Trends in Europe and the U.S.A. », Population Trends, 115, p. 24-34.

VAN OYEN H., COX B., JAGGER C., CAMBOIS E., NUSSELDER W., GILLIES Cl. et ROBINE J.-M., 2010, «Gender Gaps in Life Expectancy and Expected Years with Activity Limitations at Age 50 in the European Union: Associations with Macro-Level Structural Indicators », European Journal of Ageing , 7, p. 229-237.

VOLLSET STEIN E., 2008, «Baisse générale de la mortalité adulte en Europe de l'Ouest: les Espagnoles et les Suédoises tiennent la tête», Population et sociétés, Bulletin d'information, Ined, 450.

WEBER A., 2006, «Dépendance et des personnes âgées et handicap: les opinions des Français entre 2000 et 2005 », Études et Résultats, DREES, 49I. 